\title{
ON THE RELATION BETWEEN THE SEMIGROUP AND ITS INFINITESIMAL GENERATOR
}

\author{
FRANK NEUBRANDER
}

\begin{abstract}
We prove that for a semigroup generator $A$ the solutions of $u^{\prime}(t)=A u(t)$ and $u(0)=x$ are completely determined by the resolvent of $A$ along an equidistant sequence $\left(s_{0}+n r\right)_{n \in \mathbf{N}}, r \in \mathbf{R}, s_{0} \in \mathbf{C}$, of points in the resolvent set of $A$.
\end{abstract}

Most of the fundamental concepts in the theory of strongly continuous semigroups of bounded linear operators on a Banach space $E$ are based on the relation between the semigroup and the resolvent of its infinitesimal generator. For a given semigroup $(T(t))$ the resolvent $R(\lambda, A)$ of the infinitesimal generator $A$,

$$
A x=\lim _{t \rightarrow 0} \frac{1}{t}(T(t) x-x) \quad \text { for } x \in D(A),
$$

is given as the Laplace transform of the semigroup $(T(t))$, i.e.

$$
R(\lambda, A) x=\int_{0}^{\infty} e^{-\lambda t} T(t) x d t \quad \text { for every } x \in E
$$

and every $\lambda \in \mathbf{C}$ with

$$
\begin{aligned}
\operatorname{Re} \lambda>w_{1}:= & \inf \{w: \text { for every } x \in D(A) \text { there exist a constant } M(x, w) \\
& \text { such that } \left.\|T(t) x\| \leq M(x, w) e^{w t} \text { for every } t \geq 0\right\} \\
= & \inf \left\{\operatorname{Re} \lambda: \int_{0}^{\infty} e^{-\lambda t} T(t) x d t \text { exists for all } x \in E\right\}
\end{aligned}
$$

(see $[4])$.

From the point of view of applications to partial differential equations it is more relevant to obtain the solutions $T(\cdot) x$ of the initial value problem

$$
u^{\prime}(t)=A u(t), \quad u(0)=x \in D(A)
$$

from the resolvent of the infinitesimal generator $A$. Well-known formulas are the Yosida approximation

$$
T(t) x=\lim _{\lambda \rightarrow \infty} e^{t \lambda A R(\lambda, A)} x \quad(\text { for } x \in E)
$$

and the exponential (or Post-Widder inversion-) formula

$$
\begin{aligned}
T(t) x & =\lim _{n \rightarrow \infty}\left[\frac{n}{t} R\left(\frac{n}{t}, A\right)\right]^{n} x \\
& =\lim _{n \rightarrow \infty}(-1)^{n} \frac{1}{k !}\left(\frac{n}{t}\right)^{n+1} R^{(n)}\left(\frac{n}{t}, A\right) x \quad(x \in E) .
\end{aligned}
$$

Received by the editors October 31, 1984 and, in revised form, March 28, 1986.

1980 Mathematics Subject Classification (1985 Revision). Primary 47D05; Secondary 34G10. 
In both formulas, for $\lambda \geq \lambda_{0}$, the powers of $R(\lambda, A)$ have to be known. However, in applications it is for the most part impossible to estimate the powers of the resolvent. For practical purposes the most useful representation of $T(\cdot) x, x \in D(A)$, is given by

$$
T(t) x=\frac{1}{2 \pi i} \lim _{r \rightarrow \infty} \int_{\gamma-i r}^{\gamma+i r} e^{\lambda t} R(\lambda, A) x d \lambda \quad(t>0)
$$

the inversion formula of the Laplace transform. Investigating semigroups with different continuity properties, E. Hille and R. Phillips assumed-in analogy to the inversion formula for the general real Laplace-Stieltjes transform-the constant $\gamma$ to be greater than $\max \left\{0, w_{0}\right\} ; w_{0}$ being the growth bound of the semigroup (see [2, p. 349]). We remark that for strongly continuous semigroups the condition $\gamma>\max \left\{0, w_{0}\right\}$ can be replaced by $\gamma>w_{1}$ (the proof given by Widder $[6$, p. 64ff] carries over to the infinite-dimensional case, see $[3]$ ). For eventually normcontinuous semigroups on Banach spaces (as there are compact, differentiable, holomorphic, nilpotent or normcontinuous semigroups) [1], and for positive semigroups on Banach spaces ordered by a normal and generating cone [4], the growth bound $w_{1}$ is equal to the spectral bound of $A$ i.e. the equality

$$
s(A):=\sup \{\operatorname{Re} \lambda, \lambda \in \sigma(A)\}=w_{1}
$$

holds. Therefore, in these cases $T(t) x, x \in D(A)$, can be completely represented in terms of the infinitesimal generator $A(R(\lambda, A) x$ has to be known for $\lambda \in \gamma+i \mathbf{R}, \gamma>$ $s(A))$.

In this note we would like to call attention to a representation formula due to E. Phragmén [5, p. 360ff] or $[\mathbf{7}$, p. 166]. Using this formula we will see that the solutions of $(*)$ are completely determined by the resolvent along an equidistant sequence $\left(s_{0}+n r\right)_{n \in \mathbf{N}}, r \in \mathbf{R}, s_{0} \in \mathbf{C}$, of points in the resolvent set of $A$.

PhragmÉn's RePRESENTATION THEOREM. Let $A$ be the generator of $a$ strongly continuous semigroup $(T(t))$ on a Banach space $E$. Then, for every $x \in$ $D(A)$,

$$
T(t) x=\lim _{\lambda \rightarrow \infty} \lambda \sum_{n=1}^{\infty}(-1)^{n-1} \frac{1}{(n-1) !} e^{n \lambda t} R(n \lambda, A) x .
$$

ProOF. For $\lambda \geq w>w_{0}$ the resolvent $R(\lambda, A)$ is bounded. Therefore, for every $x \in E$,

$$
y:=\sum_{n=1}^{\infty}(-1)^{n+1} \frac{1}{n !} e^{n \lambda t} R(n \lambda, A) x
$$

exists. By

$$
R(n \lambda, A) x=\int_{0}^{\infty} e^{-n \lambda s} T(s) x d s
$$

we obtain

$$
y=\sum_{n=1}^{\infty}(-1)^{n+1} \frac{1}{n !} e^{n \lambda t} \int_{0}^{\infty} e^{-n \lambda s} T(s) x d s .
$$

Define

$$
f_{N}(s):=\left[\sum_{n=1}^{N}(-1)^{n+1} \frac{1}{n !} e^{n \lambda(t-s)}\right] T(s) x .
$$


Then, as $N \rightarrow \infty, f_{N}(\cdot)$ converges uniformly on compacts to

$$
\left(1-e^{-e^{\lambda(t-s)}}\right) T(s) x .
$$

Hence

$$
\begin{aligned}
& \int_{0}^{T}\left(1-e^{-e^{\lambda(t-s)}}\right) T(s) x d s \\
& \quad=\sum_{n=1}^{\infty}(-1)^{n+1} \frac{1}{n !} e^{n \lambda t} \int_{0}^{T} e^{-n \lambda s} T(s) x d s=: z_{T}
\end{aligned}
$$

By

$$
\left\|z_{T}-y\right\| \leq\left(\sum_{n=1}^{\infty} \frac{1}{n !} e^{n \lambda t}\right) \int_{T}^{\infty}\left\|e^{-\lambda s} T(s) x\right\| d s \rightarrow 0
$$

as $T \rightarrow \infty$ we obtain $y=\int_{0}^{\infty}\left(1-e^{-e^{\lambda(t-s)}}\right) T(s) x d s$. Now, for $0<\delta<t$,

$$
\begin{aligned}
\left\|y-\int_{0}^{t} T(s) x d s\right\| \leq & \left\|\int_{0}^{t-\delta} e^{-e^{\lambda(t-s)}} T(s) x d s\right\|+\int_{t-\delta}^{t+\delta}\|T(s) x\| d s \\
& +\left\|\int_{t+\delta}^{\infty}\left(1-e^{-e^{\lambda(t-s)}}\right) T(s) x d s\right\| .
\end{aligned}
$$

Given $\varepsilon>0$ chose $\delta$ such that the second term is less than $\varepsilon / 3$. For all $0 \leq s \leq t-\delta$ and for all $\lambda \geq \lambda_{0}$ we have

$$
\left\|\int_{0}^{t-\delta} e^{-e^{\lambda(t-s)}} T(s) x d s\right\| \leq \int_{0}^{t-\delta} e^{-e^{\lambda_{0} \delta}}\|T(s) x\| d s .
$$

Choose $\lambda_{0}>\max (w, 0)$ such that the first term of the inequality is less than $\varepsilon / 3$ for all $\lambda \geq \lambda_{0}$. Let $r>0$. Then $1-e^{-r}<r$. Therefore

$$
\begin{aligned}
\left\|\int_{t+\delta}^{\infty}\left(1-e^{-e^{\lambda(t-s)}}\right) T(s) x d s\right\| & \leq \int_{t+\delta}^{\infty} e^{\lambda(t-s)}\|T(s) x\| d s \\
& \leq M\|x\|(\lambda-w)^{-1} e^{w(t+\delta)-\lambda \delta} .
\end{aligned}
$$

Chose $\lambda_{1} \geq \lambda_{0}$ such that the third term of the inequality is less than $\varepsilon / 3$ for all $\lambda>\lambda_{1}$. Hence, for every $x \in E$,

$$
\int_{0}^{t} T(s) x d s=\lim _{\lambda \rightarrow \infty} \sum_{n=1}^{\infty}(-1)^{n+1} \frac{1}{n !} e^{n \lambda t} R(n \lambda, A) x .
$$


Now let $x \in D(A)$. Then, by $R(\lambda, A) A x=\lambda R(\lambda, A) x-x$, we obtain

$$
\begin{aligned}
T(t) x= & x+\int_{0}^{t} T(s) A x d s \\
= & x+\lim _{\lambda \rightarrow \infty} \sum_{n=1}^{\infty}(-1)^{n+1} \frac{1}{n !} e^{n \lambda t}[n \lambda R(n \lambda, A) x-x] \\
= & x+\lim _{\lambda \rightarrow \infty}\left(e^{-e^{\lambda t}}-1\right) x \\
& +\lim _{\lambda \rightarrow \infty} \lambda \sum_{n=1}^{\infty}(-1)^{n-1} \frac{1}{(n-1) !} e^{n \lambda t} R(n \lambda, A) x \\
= & \lim _{\lambda \rightarrow \infty} \lambda \cdot \sum_{n=1}^{\infty}(-1)^{n-1} \frac{1}{(n-1) !} e^{n \lambda t} R(n \lambda, A) x .
\end{aligned}
$$

Replacing $A$ by $B=-s_{0}+A$ and $T(t)$ by $e^{-s_{0} t} T(t)$ one obtains for $x \in D(A)$, $s_{0} \in \mathbf{C}$, and $r>0$,

$$
T(t) x=r e^{s_{0} t} \lim _{\substack{k \rightarrow \infty \\ k \in \mathbb{N}}} k \sum_{n=1}^{\infty}(-1)^{n-1} \frac{1}{(n-1) !} e^{n k r t} R\left(s_{0}+n k r, A\right) x
$$

Hence the solutions of the initial value problem are completely determined by $R(\lambda, A), \lambda \in\left\{s_{0}+n r, n \geq n_{0}\right\}$.

For every $x \in E$ we have

$$
\int_{0}^{t} e^{-s_{0} r} T(r) x d r=\lim _{\substack{k \rightarrow \infty \\ k \in \mathbb{N}}} \sum_{n=1}^{\infty}(-1)^{n+1} \frac{1}{n !} e^{n k r t} R\left(s_{0}+n k r, A\right) x .
$$

Using this equation and the fact that $R(\lambda, A) x$ is given as the Laplace transform of $T(t) x$ we get the following corollary.

COROLlARY. Let $A$ be the generator of a strongly continuous semigroup $(T(t))$ on a Banach space $E$ and let $Y$ be a closed supspace of $E$. Then the following statements are equivalent:

(i) $Y$ is an invariant subspace of $(T(t))$.

(ii) There exists an $s_{0} \in \mathbf{C}$, and $r \in \mathbf{R}$, and an $n_{0} \in \mathbf{N}$ such that $Y$ is an invariant subspace of $R\left(s_{0}+n r, A\right)$ for every $n \geq n_{0}$.

\section{REFERENCES}

1. E. Davis, One-parameter semigroups, Academic Press, New York, 1980.

2. E. Hille and R. S. Phillips, Functional analysis and semigroups, Amer. Math. Soc. Colloq. Publ., vol. 31, Providence, R. I., 1957.

3. F. Neubrander, Well-posedness of higher order abstract Cauchy problems, Dissertation, Tuebingen, 1984. 
4. _ Laplace transform and asymptotic behavior of strongly continuous semigroups, Houston $\mathrm{J}$. Math. (to appear).

5. E. Phragmén, Sur une extension d'un théorème classique de la théorie des fonctions, Acta Math. 28 (1904), 351-368.

6. D. V. Widder, The Laplace transform, Princeton Univ. Press, Princeton, N. J., 1946.

7. K. Yosida, Functional analysis, Grundlehren Math. Wiss., Band 123, Springer-Verlag, Berlin and New York, 1978.

Department of Mathematics, Georgetown University, Washington, D.C. 20057 